\title{
UNIVALENT FUNCTIONS WITH UNIVALENT DERIVATIVES. II
}

\author{
BY
}

S. M. SHAH( $\left.{ }^{1}\right)$ AND S. Y. TRIMBLE

1. Introduction. Let $D$ denote the open unit disc with center at the origin. It is known that if $f$ and all its derivatives are univalent in $D$, then $f$ must be an entire function of exponential type [11]. However, to conclude that $f$ is entire, we shall show it is not necessary to suppose that each derivative is univalent in $D$.

Let $\rho_{n}$ be the largest number with the property that $f^{(n)}$ is univalent in an open disc about the origin of radius $\rho_{n}$. (Note that $\rho_{n}$ is finite unless $f^{(n)}(z)=a z+b$. We shall exclude this possibility by always assuming that $f$ is not a polynomial.) In this paper, we investigate the relation between the growth of $\left\{\rho_{n}\right\}_{n=0}^{\infty}$ and the radius of convergence of $f$ about the origin. In particular, we show that if $\rho_{n}$ converges to zero slowly enough, then $f$ must still be an entire function. (See the corollary to Theorem 1.) Further, if $f$ is entire, we exhibit relations between the growth of $\left\{\rho_{n}\right\}_{n=0}^{\infty}$ and the order and type of $f$.

It is well known [12, p. 212], [3] that if $f$ is defined in a disc about the origin of radius $\rho$ by

$$
f(z)=\sum_{n=0}^{\infty} a_{n} z^{n}
$$

and if $a_{1} \neq 0$, then $f$ is univalent in this disc if

$$
\sum_{n=2}^{\infty} n\left|a_{n}\right| \rho^{n-1} \leqq\left|a_{1}\right|
$$

It is also well known [6, p. 213], [4, p. 3] that if $f$ is univalent in $D$, then $a_{1} \neq 0$ and

$$
\left|a_{2}\right| \leqq 2\left|a_{1}\right| \text {. }
$$

We shall use both these facts in the proofs below. For convenience of notation, we shall sometimes write $f^{(0)}=f$ and $a_{n}=a(n)$.

2. Derivatives with varying radii of univalence. We single out two kinds of functions. Let $f$ be an analytic function defined in $|z|<R$. (We allow $R=\infty$.) We shall say that $f$ has property $(A)$ at $N$ if there is a nonnegative integer, $N$, such that for $n \geqq N, \rho_{n}>0$. Note that this implies that if $n \geqq N$, then $a_{n+1} \neq 0$. We shall say that $f$ has property (B) at $N$ if there is a positive integer, $N$, such that

Presented to the Society, January 24, 1969; received by the editors February 18, 1969.

${ }^{(1)}$ The research work of the first author was supported by the National Science Foundation under Grant GP-7544. 
$\left\{\left|a_{n-1} / a_{n}\right|\right\}_{n=N}^{\infty}$ is a positive and nondecreasing sequence. Note that property (B) implies property (A).

THEOREM 1. Let $f$ be defined by $f(z)=\sum_{k=0}^{\infty} a_{k} z^{k}$. Let $R$ denote the radius of convergence of $f$ and $\rho_{n}$ the radius of univalence of $f^{(n)}$. Then

$$
\liminf _{n \rightarrow \infty} n \rho_{n} \leqq 4 R
$$

and

$$
R \log 2 \leqq \limsup _{n \rightarrow \infty} n \rho_{n} .
$$

If $f$ has property (A) at $N$, then

$$
\liminf _{n \rightarrow \infty}\left[n\left(\rho_{N} \rho_{N+1} \cdots \rho_{n}\right)^{1 / n}\right] \leqq 4 e R .
$$

If $f$ has property $(\mathrm{B})$, then

$$
R \log 2 \leqq \liminf _{n \rightarrow \infty} n \rho_{n} \leqq \limsup _{n \rightarrow \infty} n \rho_{n} \leqq 4 R .
$$

Proof. If an infinite number of the $\rho_{n}$ are zero, then (2.1) is obviously true. So, suppose $f$ has property (A) at $N$. Let $F_{n}(z)=f^{(n)}\left(\rho_{n} z\right)$. Using (1.2) on $F_{n}$ and assuming $n \geqq N$, we have

$$
\left|a_{n+2}\right| \leqq 4\left|a_{n+1}\right| /(n+2) \rho_{n} .
$$

Since $\lim \inf _{n \rightarrow \infty}\left|a_{n} / a_{n+1}\right| \leqq R,(2.1)$ is established. If $f$ has property (B), then $\lim _{n \rightarrow \infty}\left|a_{n}\right| a_{n+1} \mid=R$ and the right-hand part of (2.4) is established.

Using (2.5), an induction argument shows that if $k \geqq N+2$, then

So,

$$
\left|a_{k}\right| \leqq \frac{4^{k-N-1}\left|a_{N+1}\right|(N+1) !}{\left(\rho_{N} \rho_{N+1} \cdots \rho_{k-2}\right) k !} .
$$

$$
\frac{1}{R}=\limsup _{k \rightarrow \infty}\left|a_{k}\right|^{1 / k} \leqq \frac{4 e}{\lim \inf _{k \rightarrow \infty}\left[k\left(\rho_{N} \cdots \rho_{k}\right)^{1 / k}\right]} .
$$

This proves (2.3).

We no longer assume that $f$ has property (A). Let $0<r<R$. Since $\sum\left|a_{n}\right| r^{n}<\infty$, there is an increasing sequence, $\left\{n_{p}\right\}_{p=1}^{\infty}$, of positive integers such that for $p=1,2, \ldots$, and $k=2,3, \ldots$, we have $\left|a\left(n_{p}+1\right)\right| \geqq\left|a\left(n_{p}+k\right)\right| r^{k-1}$. For $n=1,2, \ldots$, let $x_{n}=$ $n\left(1-2^{-1 /(n+2)}\right)$. Then

$$
\begin{aligned}
\sum_{k=2}^{\infty} \frac{\left(k+n_{p}\right) !\left|a\left(n_{p}+k\right)\right| r^{k}\left[x\left(n_{p}\right)\right]^{k}}{(k-1) ! n_{p}^{k}} & \leqq r\left|a\left(n_{p}+1\right)\right| \sum_{k=2}^{\infty} \frac{\left(k+n_{p}\right) !}{(k-1) !}\left(\frac{x\left(n_{p}\right)}{n_{p}}\right)^{k} \\
& =\frac{\left(n_{p}+1\right) !\left|a\left(n_{p}+1\right)\right| r x\left(n_{p}\right)}{n_{p}} .
\end{aligned}
$$

Define $F_{p}$ in $D$ by

$$
F_{p}(z)=f^{\left(n_{p}\right)}\left(r z x\left(n_{p}\right) / n_{p}\right) .
$$


From (1.1) and (2.6), it follows that $F_{p}$ is univalent in $D$. Hence, $r x\left(n_{p}\right) / n_{p} \leqq \rho\left(n_{p}\right)$. Since $\lim _{n \rightarrow \infty} x_{n}=\log 2,(2.2)$ is proved.

Now assume that $f$ has property (B) at $N$. If $n \geqq N$, let $r_{n}=\left|a_{n-1} / a_{n}\right|$. Then for $k=2,3, \ldots$, we have $\left|a_{n+1}\right| \geqq\left|a_{n+k}\right| r_{n}^{k-1}$. Using the preceding argument, it follows that if $n \geqq N$, then

$$
r_{n} x_{n} \leqq n \rho_{n}
$$

Since $\lim _{n \rightarrow \infty} r_{n} x_{n}=R \log 2$, (2.4) follows and the entire theorem is proved.

COROLlaRY. If $\lim _{n \rightarrow \infty} n \rho_{n}=\infty$, then $f$ is a transcendental entire function. If $f$ is a transcendental entire function, then $\lim \sup _{n \rightarrow \infty} n \rho_{n}=\infty$. If $f$ is a transcendental entire function with property (B), then $\lim _{n \rightarrow \infty} n \rho_{n}=\infty$.

The converse of the first part of this corollary is false. In fact, if $\left\{b_{n}\right\}_{n=0}^{\infty}$ is any sequence of positive numbers such that $\lim _{\inf _{n \rightarrow \infty}} b_{n}>0$, then there is an entire function, $f$, such that each $\rho_{n}>0$ but $\lim _{\inf _{n \rightarrow \infty}} b_{n} \rho_{n}=0$. For instance, consider the following: If $n=0,1, \ldots$, let $a_{2 n}=1 /((2 n) !)^{1 / 2}$ and $a_{2 n+1}=1 / b_{2 n}((2 n+1) !)^{1 / 2}$. (If $b_{2 n}=0$, let $a_{2 n+1}=0$. There can only be a finite number of these.) Define $f_{1}$ by $f_{1}(z)=\sum_{n=0}^{\infty} a_{n} z^{n}$. Then $f_{1}$ is certainly entire, and for large $n,(2.5)$ becomes $b_{2 n} \rho_{2 n} \leqq 4 /(2 n+2)^{1 / 2}$.

The converse of the second part of the corollary is also false. Let $n_{1}=2$. Suppose that $p \geqq 1$ and that $n_{p}$ has been chosen. Let $n_{p+1}$ be an integer such that $n_{p+1}>n_{p}$ and if $j \geqq n_{p+1}+1-n_{p}$, then

$$
j^{2}\left(j+n_{p}\right)^{n_{p}+1} \leqq n_{p}^{(j-1) / 2} .
$$

If $n=n_{p}$ for some $p$, let $a_{n+1}=1$. Otherwise, let $a_{n}=0$. Define $f_{2}$ by $f_{2}(z)=\sum_{j=1}^{\infty} a_{j} z^{j}$. We use (1.1) to show that $f_{2}^{\left(n_{p}\right)}$ is univalent in a disc about the origin of radius $1 /\left(n_{p}\right)^{1 / 2}$ :

$$
\begin{aligned}
\sum_{j=2}^{\infty} j\left|\frac{\left(j+n_{p}\right) ! a\left(j+n_{p}\right)}{j !}\right| \frac{1}{n_{p}^{(j-1) / 2}} & \leqq \sum_{j=n_{p+1}+1-n_{p}}^{\infty} \frac{\left(j+n_{p}\right) !}{(j-1) ! n_{p}^{(j-1) / 2}} \\
& <\sum_{j=n_{p+1}+1-n_{p}}^{\infty} \frac{\left(j+n_{p}\right)^{n_{p}+1}}{n_{p}^{(j-1) / 2}} \leqq \sum_{j=2}^{\infty} \frac{1}{j^{2}}<\left(n_{p}+1\right) ! .
\end{aligned}
$$

Hence, for $f_{2}, \lim \sup _{n \rightarrow \infty} n \rho_{n} \geqq \lim \sup _{p \rightarrow \infty} n_{p} /\left(n_{p}\right)^{1 / 2}=\infty$, but the radius of convergence of $f_{2}$ about the origin is 1 .

3. Entire functions and univalent derivatives. Next, we obtain relations between the radii of univalence of the derivatives of an entire function, $f$, and the order and type of $f$. In this and the following section, we shall let $\Lambda$ be the order and $\lambda$ be the lower order of $f$. If $0<\Lambda<\infty$, we let $T$ be the type and $t$ be the lower type of $f$.

Several results connecting the order and type of an entire function with the sequence, $\left\{\rho_{n}\right\}_{n=1}^{\infty}$, already exist. Boas has shown [1] that if $f$ is a transcendental entire function of exponential type less than $\log 2$, then there is a subsequence, 
$\left\{\rho\left(n_{p}\right)\right\}_{p=1}^{\infty}$, such that $\rho\left(n_{p}\right) \geqq 1$ for all $p$. (Levinson [5] supplied a second proof of this.) Boas also pointed out [1] that if the order of $f$ is less than one, or if $f$ is of . order one but minimal type, then $\lim \sup _{n \rightarrow \infty} \rho_{n}=\infty$. Pólya $[7$, p. 18] has stated that

$$
\liminf _{n \rightarrow \infty} \frac{\log \rho_{n}}{\log n} \leqq \frac{1-\Lambda}{\Lambda} \leqq \limsup _{n \rightarrow \infty} \frac{\log \rho_{n}}{\log n}
$$

We shall improve these results and establish several more as well.

Lemma. Let $f$ be defined on $|z|<R$ by $f(z)=\sum_{k=0}^{\infty} a_{k} z^{k}$. (We allow $R=\infty$.) Let $v(r)$ denote its central index. For $n=1,2, \ldots$, let $x_{n}=n\left(1-2^{-1 /(n+2)}\right)$. Then if $R>r>0$ and $v(r) \geqq 2$,

$$
r x(v(r)-1)<(v(r)-1) \rho(v(r)-1) .
$$

Proof. Let $0<r<R$. Then $|a(v(r))|>|a(v(r)+k)| r^{k}$ for $k=1,2, \ldots$ Suppose $n=v(r)-1$. Using the same argument that proved (2.2), it follows that $r x_{n}<n \rho_{n}$.

THEOREM 2. Let $f$ be a transcendental entire function defined by $f(z)=\sum_{n=0}^{\infty} a_{n} z^{n}$. Let $\delta=\liminf _{r \rightarrow \infty} v(r) / r$. Then

$$
\begin{gathered}
\liminf _{n \rightarrow \infty} \frac{\log \left(\max \left\{1, n \rho_{n}\right\}\right)}{\log n} \leqq \frac{1}{\Lambda}, \\
\frac{1-\lambda}{\lambda} \leqq \limsup _{n \rightarrow \infty} \frac{\log \rho_{n}}{\log n}
\end{gathered}
$$

and

$$
\frac{\log 2}{\delta} \leqq \limsup _{n \rightarrow \infty} \rho_{n}
$$

Suppose that $0<\Lambda<\infty$ and that $f$ has property (A) at $N$. In this case

$$
e^{\Lambda-1} \liminf _{n \rightarrow \infty} n^{\Lambda-1} \rho_{n}^{\Lambda} \leqq \liminf _{n \rightarrow \infty}\left(\rho_{N} \rho_{N+1} \cdots \rho_{n}\right)^{\Lambda / n}\left(n^{\Lambda-1}\right) \leqq \frac{4^{\Lambda} e^{\Lambda-1}}{\Lambda T} .
$$

In any case,

$$
\liminf _{n \rightarrow \infty} n^{\Lambda-1} \rho_{n}^{\Lambda} \leqq \frac{4^{\Lambda}}{\Lambda T}
$$

Proof. To prove (3.2), we may assume that $n \rho_{n} \geqq 1$ for all $n$ and that $\Lambda>0$. It is known [8] that

$$
\frac{1}{\Lambda} \geqq \liminf _{n \rightarrow \infty} \frac{\log \left|a_{n} / a_{n+1}\right|}{\log n}
$$

From (2.5), we have that

Hence,

$$
\log n \rho_{n}<\log 4+\log \left|a_{n+1}\right| a_{n+2} \mid .
$$

$$
\frac{1}{\Lambda} \geqq \liminf _{n \rightarrow \infty} \frac{\log n}{\log (n+1)}\left(\frac{\log n \rho_{n}}{\log n}-\frac{\log 4}{\log n}\right)=\liminf _{n \rightarrow \infty} \frac{\log n \rho_{n}}{\log n}
$$


It has been shown [13] that

$$
\frac{1}{\lambda}=\limsup _{r \rightarrow \infty} \frac{\log r}{\log v(r)}
$$

Since $f$ is entire, $\lim _{r \rightarrow \infty} v(r)=\infty$. So, from (3.1), for all large $r$, we have

Hence,

$$
\frac{\log r}{\log v(r)} \leqq \frac{\log \rho(v(r)-1)}{\log (v(r)-1)}+1+\frac{\log x(v(r)-1)}{\log v(r)}
$$

This establishes (3.3).

$$
\begin{aligned}
\frac{1}{\lambda} & \leqq \limsup _{r \rightarrow \infty} \frac{\log \rho(v(r)-1)}{\log (v(r)-1)}+1 \\
& \leqq 1+\limsup _{n \rightarrow \infty} \frac{\log \rho_{n}}{\log n}
\end{aligned}
$$

Using (3.1) again, for large $r$ we have

Therefore,

$$
\frac{r}{v(r)}<\frac{\rho(v(r)-1)}{x(v(r)-1)}
$$

This proves (3.4).

$$
\frac{1}{\delta} \leqq \limsup _{r \rightarrow \infty} \frac{\rho(v(r)-1)}{x(v(r)-1)} \leqq \frac{\lim \sup _{n \rightarrow \infty} \rho_{n}}{\log 2}
$$

Now suppose that $f$ has property (A) at $N$. It is known $[2, \mathrm{p} .11]$ that

$$
e \Lambda T=\lim _{n \rightarrow \infty} \sup n\left|a_{n}\right|^{\Lambda / n} \text {. }
$$

But

From (2.5),

$$
\limsup _{n \rightarrow \infty} n\left|a_{n}\right|^{\Lambda / n}=\limsup _{n \rightarrow \infty} n \prod_{k=N+2}^{n}\left|\frac{a_{k}}{a_{k-1}}\right|^{\Lambda / n} \text {. }
$$

$$
\begin{aligned}
e \Lambda T & \leqq \limsup _{n \rightarrow \infty} n \prod_{k=N+2}^{n}\left(\frac{4}{k \rho_{k-2}}\right)^{\Lambda / n} \\
& =(4 e)^{\Lambda} \limsup _{n \rightarrow \infty} \frac{n^{1-\Lambda}}{\left(\rho_{N} \rho_{N+1} \cdots \rho_{n}\right)^{\Lambda / n}} .
\end{aligned}
$$

Since the left-hand inequality of (3.5) is always true, (3.5) is proved. If an infinite number of the $\rho_{n}$ are zero, then (3.6) is trivial. If only a finite number of the $\rho_{n}$ are zero, then (3.6) becomes (3.5). This establishes the theorem.

Suppose that $0<\Lambda<\infty$, and let $\delta^{\prime}=\liminf _{r \rightarrow \infty} v(r) / r^{\Lambda}$. In [9], it was shown that $\delta^{\prime} \leqq \Lambda t \leqq \Lambda T$. Hence, if $\Lambda=1$, (3.4) can be written as

$$
\frac{\log 2}{t} \leqq \frac{\log 2}{\delta} \leqq \limsup _{n \rightarrow \infty} \rho_{n}
$$

Further, if $\lambda<1$, then $\delta=0$, and either (3.3) or (3.4) imply that lim $\sup _{n \rightarrow \infty} \rho_{n}=\infty$. This, together with (3.8), yield improvements on the results of Boas. We note that 
there are transcendental entire functions of order one such that $\delta=0$ and $T>0$. For example, let

$$
\psi(z)=\sum_{n=1}^{\infty} \frac{z^{p_{n}}}{p_{n} !}
$$

where $p_{1}=3$ and if $n>1, p_{n}=\left[p_{n-1} \log p_{n-1}\right]$. Then $\Lambda=T=1$, but $\delta=0$. We summarize these results in a corollary.

Corollary 1. If $\lambda<1$, then $\lim \sup _{n \rightarrow \infty} \rho_{n}=\infty$. If $\Lambda=1$, then

$$
\frac{\log 2}{t} \leqq \frac{\log 2}{\delta} \leqq \limsup _{n \rightarrow \infty} \rho_{n}
$$

In particular, if $\delta=0$, then $\lim \sup _{n \rightarrow \infty} \rho_{n}=\infty$.

From (3.2) and (3.4), we get a second corollary.

Corollary 2. If $\Lambda>1$, then $\lim _{\inf _{n \rightarrow \infty}} \rho_{n}=0$. If $\Lambda=1$, then

$$
\frac{4}{T} \geqq \liminf _{n \rightarrow \infty} \rho_{n} .
$$

In particular, if $\Lambda=1$ and $T=\infty$, then $\lim \inf _{n \rightarrow \infty} \rho_{n}=0$.

If $\Lambda=1$ and $0<T<\infty$, then it may be the case that

$$
0<\liminf _{n \rightarrow \infty} \rho_{n} \leqq \limsup _{n \rightarrow \infty} \rho_{n}<\infty .
$$

The function, $\phi(z)=e^{z}$, is an example of this.

THEOREM 3. Let $f$ be an entire function with property (B) at N. Let

Then

$$
\gamma=\limsup _{r \rightarrow \infty} v(r) / r
$$

and

$$
\begin{aligned}
& \liminf _{n \rightarrow \infty} \frac{\log \rho_{n}}{\log n}=\frac{1-\Lambda}{\Lambda} \leqq \frac{1-\lambda}{\lambda}=\limsup _{n \rightarrow \infty} \frac{\log \rho_{n}}{\log n}, \\
& \frac{\log 2}{\gamma} \leqq \liminf _{n \rightarrow \infty} \rho_{n} \leqq \frac{4}{\gamma}
\end{aligned}
$$

$$
\frac{\log 2}{\delta} \leqq \limsup _{n \rightarrow \infty} \rho_{n} \leqq \frac{4}{\delta}
$$

Suppose $0<\Lambda<\infty$. Then

$$
(\log 2)^{\Lambda} \leqq \Lambda t e^{1-\Lambda} \limsup _{n \rightarrow \infty}\left(\rho_{N} \rho_{N+1} \cdots \rho_{n}\right)^{\Lambda / n}\left(n^{\Lambda-1}\right) \leqq 4^{\Lambda}
$$

and

$$
(\log 2)^{\Lambda} \leqq \Lambda T e^{1-\Lambda} \liminf _{n \rightarrow \infty}\left(\rho_{N} \rho_{N+1} \cdots \rho_{n}\right)^{\Lambda / n}\left(n^{\Lambda-1}\right) \leqq 4^{\Lambda}
$$


Proof. It is known [8] that

$$
\liminf _{n \rightarrow \infty} \frac{\log \left|a_{n}\right| a_{n+1} \mid}{\log n}=\frac{1}{\Lambda} \leqq \frac{1}{\lambda}=\limsup _{n \rightarrow \infty} \frac{\log \left|a_{n}\right| a_{n+1} \mid}{\log n} .
$$

From (2.5) and (2.7), it follows that for $n \geqq N$

$$
x_{n}\left|\frac{a_{n-1}}{a_{n}}\right|<n \rho_{n}<4\left|\frac{a_{n+1}}{a_{n+2}}\right| .
$$

Hence, from (3.12) and (3.13), we get (3.9).

Since $f$ has property (B), we have that

$$
\gamma=\limsup _{n \rightarrow \infty} n\left|\frac{a_{n}}{a_{n-1}}\right|
$$

and

$$
\delta=\liminf _{n \rightarrow \infty} n\left|\frac{a_{n}}{a_{n-1}}\right| .
$$

From this, (2.5), and (2.7), parts (3.10) and (3.11) can be proved.

Assume now that $0<\Lambda<\infty$. From [10], it follows that

$$
e \Lambda t=\liminf _{n \rightarrow \infty} n\left|a_{n}\right|^{\Lambda / n} \text {. }
$$

We use this, (3.7), and (3.13) to establish the last two parts of the theorem. The proofs are similar to the proof of (3.5), and so are omitted.

COROLlaRY. Let $f$ be an entire function with property (B).

(i) If $\Lambda<1$, then $\lim _{n \rightarrow \infty} \rho_{n}=\infty$.

(ii) If $\lambda>1$, then $\lim _{n \rightarrow \infty} \rho_{n}=0$.

(iii) If $\Lambda=1$, then $\lim _{n \rightarrow \infty} \rho_{n}=\infty$ if and only if $T=0$. If $\lim _{n \rightarrow \infty} \rho_{n}=0$, then $t=\infty$.

Proof. Parts (i) and (ii) follow from (3.9). From [9], we have that if $\Lambda=1$, then $\delta \leqq t \leqq T \leqq \gamma \leqq e T$. Part (iii) follows from this, (3.10), and (3.11).

4. Conclusion. So far, all of the work has been done with functions defined in discs centered at the origin. However, this work immediately carries over to functions defined in a disc centered at any point in the plane. To be specific, let $f$ be analytic on $\Delta=\left\{z:\left|z-z_{0}\right|<r\right\}$ and let $g(z)=f\left(r z+z_{0}\right)$ for $z \in D$. Then $f^{(n)}$ is univalent on $\left\{z:\left|z-z_{0}\right|<\rho_{n} \leqq r\right\}$ if and only if $g^{(n)}$ is univalent on $\left\{z:|z|<\rho_{n} / r\right\}$.

The authors would like to thank Professor J. D. Buckholtz for reading the manuscript and for helpful suggestions.

\section{REFERENCES}

1. R. P. Boas, Univalent derivatives of entire functions, Duke Math. J. 6 (1940), 719-721

2. —- Entire functions, Academic Press, New York, 1954.

3. A. W. Goodman, Univalent functions and nonanalytic curves, Proc. Amer. Math. Soc. 8 (1957), 598-601.

4. W. K. Hayman, Multivalent functions, Cambridge Univ. Press, Cambridge, 1958. 
5. N. Levinson, $A$ theorem of Boas, Duke Math. J. 8 (1941), 181-182.

6. Zeev Nehari, Conformal mapping, McGraw-Hill, New York, 1952.

7. G. Pólya, On the zeros of the derivatives of a function and its analytic character, Bull. Amer. Math. Soc. 49 (1943), 178-191.

8. S. M. Shah, On the lower order of integral functions, Bull. Amer. Math. Soc. 52 (1946), 1046-1052.

9. —- The maximum term of an entire series (III), Quart. J. Math. Oxford Ser. 19 (1948), 220-223.

10. - On the coefficients of an entire series of finite order, J. London Math. Soc. 26 (1951), 45-46.

11. S. M. Shah and S. Y. Trimble, Univalent functions with univalent derivatives, Bull. Amer. Math. Soc. 75 (1969), 153-157.

12. E. C. Titchmarsh, Theory of functions, Oxford Univ. Press, Oxford, 1944.

13. J. M. Whittaker, The lower order of integral functions, J. London Math. Soc. 8 (1933), 20-27.

UNIVERSITY OF KENTUCKY,

LEXINGTON, KeNTUCKY 